\title{
Effect of extracorporeal shockwave therapy on palmar fibromatosis
}

\author{
Menna Allah M. SAAD ${ }^{1 \star 凶}$, Amal M. ABD ELBAKY ${ }^{2}$, Ashraf ABO ALFOTOOH ${ }^{3}$ and Karim I. SAAFAN ${ }^{2}$ \\ ${ }^{1}$ Department of Physical Therapy for Surgery, Faculty of Physical Therapy, MTI University, Cairo, Egypt \\ ${ }^{2}$ Department of Physical Therapy for Surgery, Faculty of Physical Therapy, Cairo University, Giza, Egypt \\ ${ }^{3}$ Department of Plastic Surgery, Faculty of Medicine, Cairo University, Cairo, Egypt \\ Corresponding author's Email: mennasaad6917@gmail.com; (DORCiD: oooo-0001-9045-7175
}

\section{ABSTRACT}

Introduction. Extracorporeal shock wave therapy (ESWT) is therapeutic modality that applies shockwave from outer part of the body to a specific lesion and promotes revascularization and hence stimulates the curing process of connective tissues, relieves pain and improves function. Aim. The present study was done to detect the role of extracorporeal shock wave therapy in reducing palmar fibromatosis using Arabic version of modified QuickDASH-9 scale. Methods. Thirty patient with age ranged from 50 to 70 suffering from palmar fibromatosis were randomly selected from faculty of physical therapy modern technology and information university outpatient hand clinic, Cairo, Egypt and distributed into two equal groups: study group (A) received ESWT once a week for six weeks, done at frequency of $6-8 \mathrm{~Hz}$ with 1700 pulse/session at $0.18 \mathrm{mj} / \mathrm{cm} 2$ with traditional physical therapy protocol (U.S, massage, stretching ex, range of motion (ROM) ex and splinting). Group B received traditional physical therapy protocol. The measurements were conducted pre and post six weeks of treatment using Arabic version of Modified Quick DASH-9 scale and Jamar hand dynamometer. The study was carried out from April to December 2020. Descriptive statistics and unpaired t-test were conducted for comparison of age between groups. Results. There was a significant decrease in the score of Arabic modified Quick DASH scoring which is a good sign of improvement and increase in the hand grip strength. Conclusion. Extracorporeal shockwave is a beneficial method in reducing the progress of palmar fibromatosis.

\author{
Original Article \\ PII: S225199392100002-11 \\ Rec. 21 November 2020 \\ Rev. 17 January 2021 \\ Acc. 25 January 2021
}

\section{Keywords}

Extracorporeal shock wave therapy (ESWT), Shockwave Therapy, Palmar fibromatosis, Arabic Modified Quick DASH-9 scale,

Jamar hand

dynamometer

\section{INTRODUCTION}

Palmar fibromatosis is a disease which develops gradually in stages as a result of thickening of palmar facia that provides cords and nodules and it reveals loss or decrease in ROM of the involved fingers and pain with tenosynovitis. Male people are the most affected, more than 50 years old with different and un known factors (liquor addiction, smoking, thyroid issues, liver illness, diabetes, past hand injury, epilepsy) and with certain hereditary factors. Palmar fibromatosis is diagnosed by fibrotic stage that generate from fibrocellular proliferation. The most affected finger normally is the ring finger then the little and center fingers (Figure 1 ) Can make certain tasks more difficult of day living, for example, face washing, brushing hair and placing hand in a glove and shaking hands [1].

The inception of palmar fibromatosis is yet unclear, although there are various physicians that specialists have gone over consistently. Generally medical procedure has been the main therapy for palmar fibromatosis however it's not the only one [2]. Numerous non-surgical treatments have been explored for the therapy of palmar fibromatosis, needle fasciotomy, nonstop gradual skeletal traction, radiation, dimethyl sulfoxide, vitamin E, steroid infusions, radiation, interferon and enzymatic fasciotomy. A large portion of these treatments have no longer confirmed or demonstrated to be clinically valuable. After all, Shockwave treatment has shown empowering results [3]. Finding is typically based on signs and symptoms; Patients, at first, may be symptomless and they may introduce withdrawal of palmar facia related with nodules, at a later stage their capacity to hold and maintain items plus ache and pain logically decline [4].

Extracorporeal shockwave treatment (ESWT) as a noninvasive treatment is utilizing acoustic waves described by a sharp, unexpected, and quick change in pressure factor as a wave front with a speed higher than the speed of sound followed by a more drawn out negative tail to get a body reaction. Since the principal clinical report performed on kidney stone destruction goal by high excessive electrohydraulic ESWT on Dec 13, 1980 in 
the Lancet by Chaussy et al. [5] in palmar fibromatosis, just two ongoing case arrangement have been accounted for either high vivacious centered ESWT in four patients [6] or radial ESWT in a solitary case [7] with no long term follow it was conjectured comparative consequences for palmar fibromatosis in decreasing contracture and pain. ESWT has more extensive and more secure than medical procedures with minimal effort in correlation with medical procedures. Taking into account that palmar fibromatosis contractures are shallow, we speculated that a treatment with ESWT was compelling to decrease them and improve hand work.
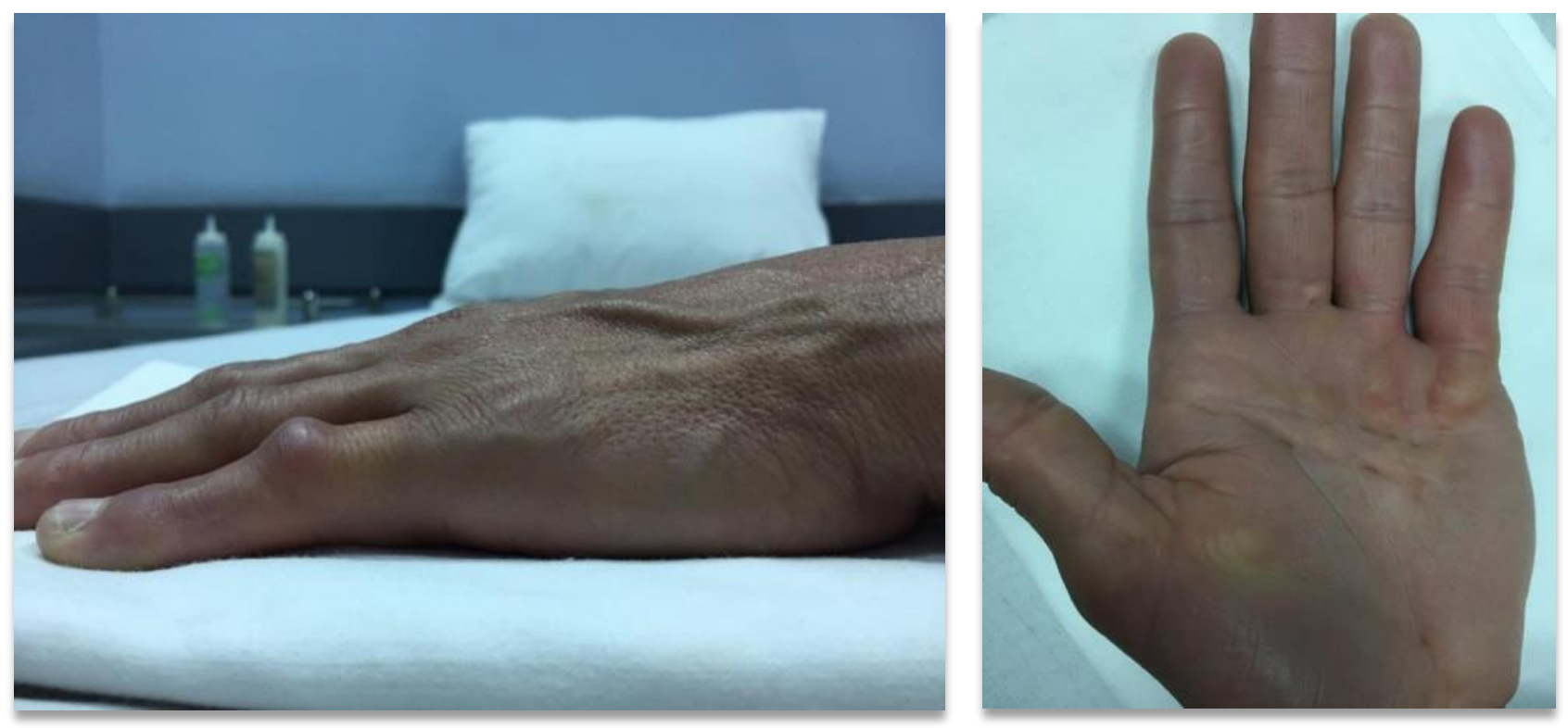

Figure 1. Palmar fibromatosis.

\section{MATERIAL AND METHODS}

Thirty patients who diagnosed with palmar fibromatosis of stage $\mathrm{N}$ \& one according to staging system of tubiana classification, with age ranged from 50 to 70 years have been selected for this study. The participants have been selected from faculty of physical therapy modern technology and information university outpatient hand clinic Cairo, Egypt. The study was conducted from April 2020 to December 2020, and participants were divided randomly into 2 groups: study group (A) that was composed of 15 patients who received ESWT once a week for six weeks, done at a frequency of $6-8 \mathrm{~Hz}$ with 1700 pulse/session at $0.18 \mathrm{mj} / \mathrm{cm} 2$ with traditional physical therapy protocol (U.S, massage, stretching ex, ROM ex and splinting). Group B that was composed of 15 patients who received traditional physical therapy protocol (U.S, massage, stretching ex, ROM ex and splinting), twice a week for 6 weeks.

\section{Ethical approval}

The protocol of this study was approved by the ethical committee board of the Faculty of Physical Therapy Cairo University; Giza, Egypt [No. P.T.REC/012/002688]. Every participant applied informed consent before starting the study. All participants were informed about the nature and the effect of the treatment and measurement tools. The Participants were also instructed to report any side effects during the treatment protocol like itching etc.

\section{Inclusive and Exclusion criteria}

Inclusive criteria was included of patients with palmar fibromatosis are presented to our out faculty of physical therapy modern technology and information university outpatient hand clinic were signed in the study, based on the following inclusion criteria: 1) Diagnosis of palmar fibromatosis in a minimum of one finger; 2) Patients who were able to attend the follow up visites; 3) Age ranged from 50 to 70 years of age; 4) Those with a flexion of 30 degrees or less in the metacarpophalangeal (MP) joint.

Exclusion criteria were included: A) Patient who had any neurological, neuromuscular and chronic disorder affect the hands; B) Patients who did not accept treatment; C) Patients who were contraindicated for the ESWT; D) Loss of extension in the involved the metacarpophalangeal (MP), proximal interphalangeal (PIP) and Distal interphalangeal (DIP) joints. 


\section{The application of treatment method}

All patients were applied the table top test. The extracorporeal shockwave treatment (ESWT) application was performed with the patient sitting on a gurney with the elbow flexed at 30 degrees, the forearm in supination and the probe of the ESWT device positioned 90 degrees tangent to the pain site. Povidone-iodine solution was used for cleaning the application site and gel was applied on the skin for conductivity. There was one no any local anesthesia applied for patient. The regions with nodules were marked and the probe was moved around this mark in a circular fashion. Same physiotherapist performed the treatment using the ESWT device (gymna code: SN 4300431 made in USA) once a week for six weeks, at a frequency of 6 to $8 \mathrm{~Hz}$ with $1700 \mathrm{pulse} / \mathrm{session}$ at $0.18 \mathrm{~mJ} / \mathrm{cm} 2$ [8] (Figure 2).

The physiotherapist and the patient had to wear earmuffs because of high noise of the operation device. The application of hot or cold pack after the treatment is not allowed following ESWT application. None of the patients reported the rarely seen of redness, pain or swelling. The patients were evaluated with Arabic modified Quick DASH-9 questionnaire and their grip strength were measured using a Jamar dynamometer. Grip strength of the patients was measured three times with one minute intervals between measurements and the average of the three measurements was noted.

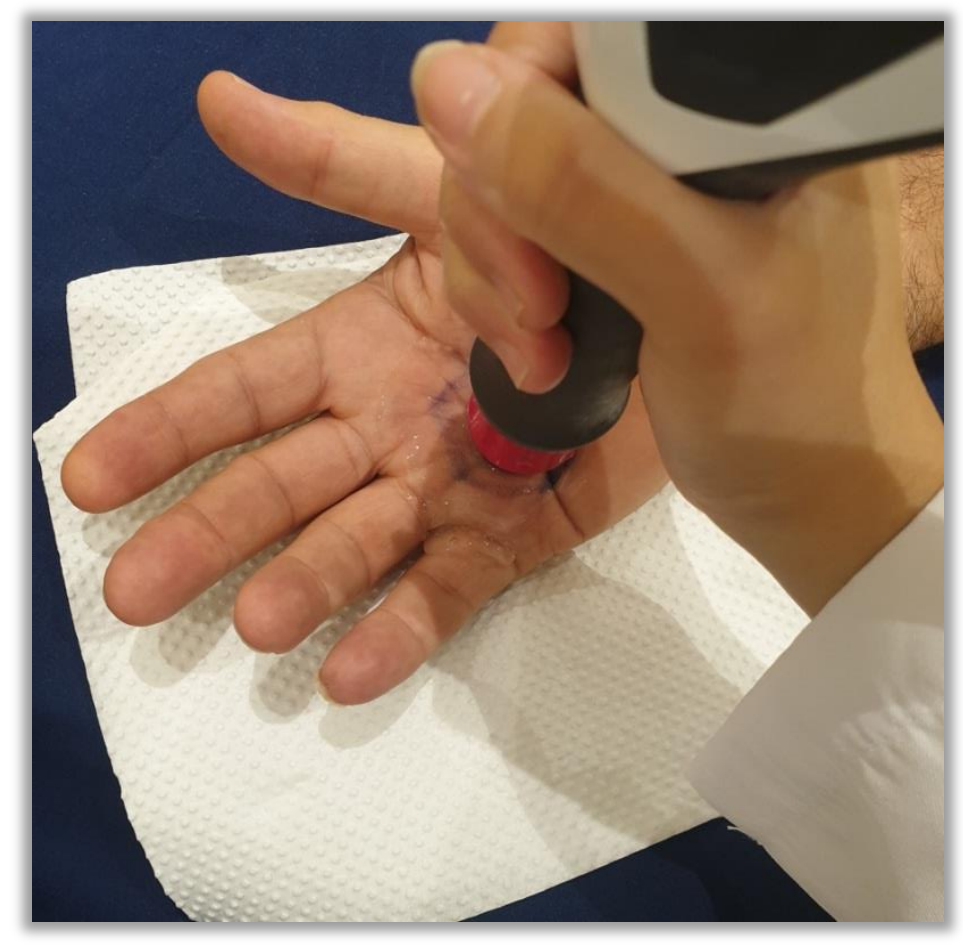

Figure 2. Application of extra corporeal shock wave therapy.

\section{Measurements}

\section{Arabic modified Quick DASH} questionnaire. The Arabic Modified Quick DASH-9 is consisted of the following: A) Brief instructions that informed the patients about how to do the questionnaire; B) The point number (1-6) that are about the degree of hardness in doing various physical activities related to the arm, shoulder or hand problems; C) The two points (7 and 8) are about the impact of upper extremities problems in the social activities; D) Point number 9 as about arm, shoulder or hand pain; E) Each point as five response scores, ranging from no problem to unable to perform activity (0-4) Likert scale; F) The Arabic modified Quick DASH-9 allows for one missing response.

The Arabic Modified Quick DASH-9 Score = [(sum) *1.1]*5/2, a missing response is added as the average of the remaining [9] (Figure 3).

\section{QuickDASH-9}

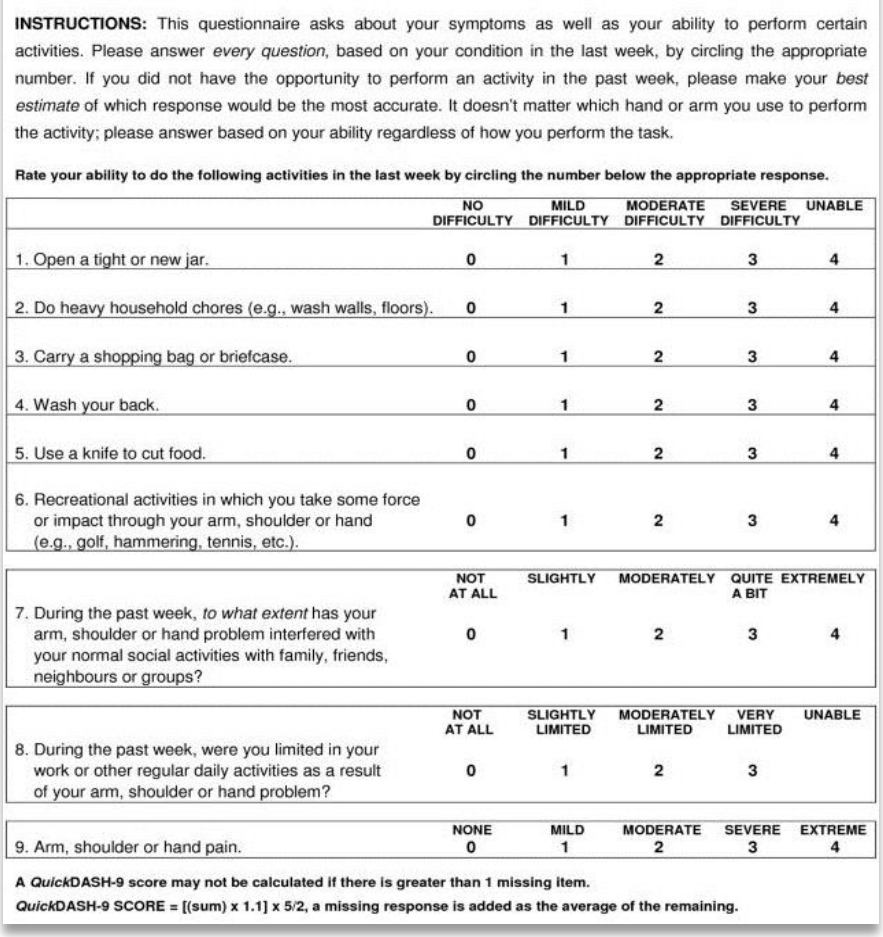

Figure 2. Modified QuickDASH-9 scale. 
Jamar hand grip dynamometer. Therapeutic grip strength was measured by using a Jamar hand grip dynamometer with one-minute intervals between measurements and the average of the three measurements was noted, the patient position was as sitting with shoulder adduction. The elbow was flexed to $90^{\circ}$ and the forearm was in neutral rotation. The wrist was at neutral to $30^{\circ}$ extension while gripping. The patient was asked to grip with full power of force for at least 2 seconds for full muscle recruitment. The therapist provided verbal words (i.e., "Ready? Steady, go! More, Much more!") until grip strength increased to the top, then said, "enough stop" grip strength was recorded, rounded to the nearest pound and the sign has been returned to zero [10] (Figure 4).

Figure 4. Measurment of hand grip using jamar hand grip dynamometer.

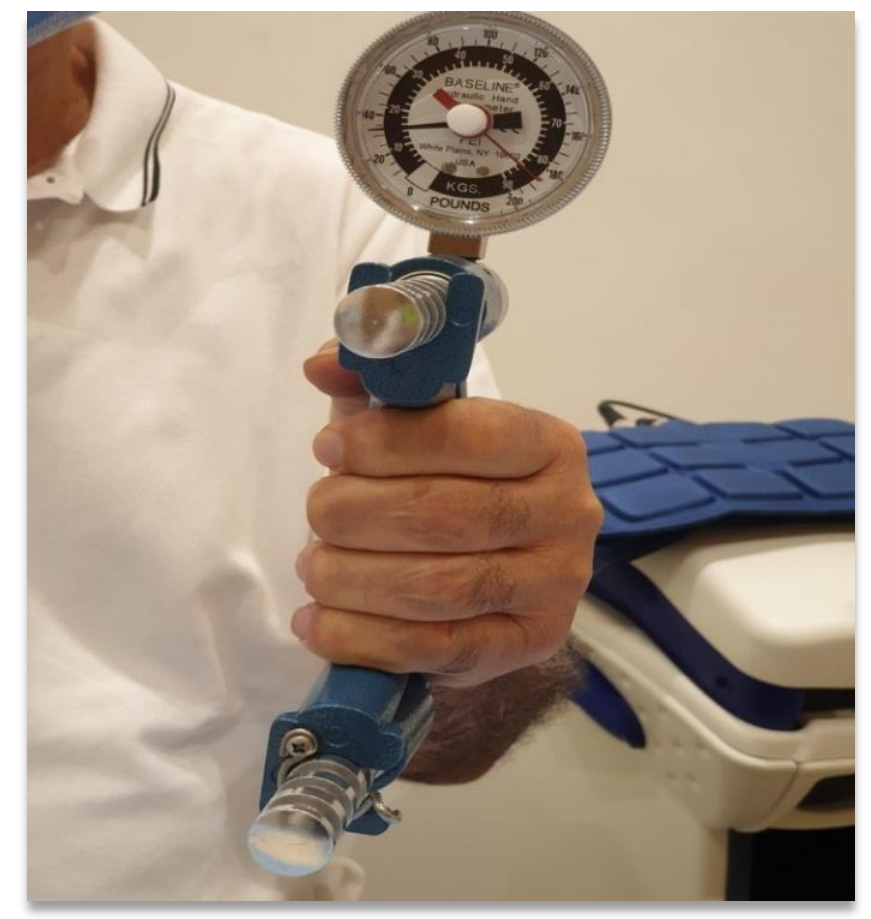

\section{Treatment procedures}

Study group (A). Patient treated as outpatient was given information about the measurement and treatment procedures as well as about the extracorporeal shockwave before the beginning of the treatment, was asked to follow and physical therapist instruction like wearing the splint and doing daily stretching, and was asked to avoid predisposing factors as any trauma to the affected hand.

Groupe B (traditional physical therapy protocol). Only received two sessions per week for 6 weeks involved ultrasound, massage splinting and stretching techniques: Low-intensity pulsed ultrasound (LIPUS) is a sort of ultrasound that produced at a way lower intensity $\left(<3 \mathrm{~W} / \mathrm{cm}^{2}\right)$ than traditional ultrasound energy and output within the mode of pulse wave, and it's typically used for therapeutic purpose in rehabilitation treatment, patient position sitting with forearm supination elbow flexion 30 degree and the probe of the ultrasound was perpendicular at the site of the contracture at pulsed mode with intensity lower than $3 \mathrm{w} / \mathrm{cm} 2$ at circular motion for 5 min every session [11].

\section{Splinting}

Thermoplastic palmar is totally based finger extension supports, developed with Velcro fastenings. The patients were trained to wear the supports during rest around evening time. The braces were remolded as upgrades were made [12].

\section{Massage therapy}

1) Before extending, the hand must be in warm water or a warm pack; 2) Intensely scented creams were kept away from; 3) Creams with hydrocarbons and synthetic inflammation substances have been additionally avoided; 4) A characteristic plant-based lotion with common calming properties was utilized;

4) Rub the palms every which way on particularly around the thickening 5 min each meeting [13].

\section{Stretching}

1) Gently twist the fingers in reverse from the palm; 2) Placing fingers on the edge of a table, palm down, and afterward lift the palm upward slowly, as you keep your fingers level on the table; 3) The wrist should be moved from side to side; 4) Putting his/her hand in a petition position pushing the palms and fingers together as home program and little change in way of life; 5) Don't over stretch the wrist and fingers which might be counter beneficial [14].

\section{Statistical analysis}

Descriptive statistics and unpaired t-test were done for comparison of age between groups. Fisher exact was carried out for comparison of sex distribution between groups. Normal distribution of data was checked using the Shapiro-Wilk test for all variables. Levene's test for homogeneity of variances was conducted to test 
the homogeneity between groups. Unpaired t-test was conducted to compare the mean values of regarding Modified Arabic DASH-9 scorring and hand grip strength between the groups A and B. Paired t-test was conducted for comparison between pre and post treatment in each group. The level of significance for all statistical tests was set at $\mathrm{p}<0.05$. All statistical analysis was conducted through the statistical package for social studies (SPSS) version 25 for windows (IBM SPSS, Chicago, IL, USA).

\section{RESULTS}

\section{Subject characteristics}

Table 1 showed the subject characteristics of the groups A and B. There was no significant difference between groups in age and sex distribution ( $\mathrm{P}>0.05)$.

\section{Effect of treatment on Modified Arabic DASH score and hand grip strength}

Within group comparison. There was a significant decrease in modified Arabic DASH-9 scoring post treatment in the group $A$ and $B$ compared with that pretreatment $(\mathrm{P}<0.001)$. The percent of decrease in Modified Arabic DASH score in the group A was 34.68\% and that for group B were $11.17 \%$. There was a significant increase in hand grip strength post treatment in the groups $A$ and $B$ compared with that pretreatment $(P<0.001)$. The percent of increase in hand grip strength in group A were 30.07 while that in group B was 10.73\% (Table 2).

Between groups comparison. There was no significant difference between groups pre-treatment $(P>0.05)$. Comparison between the groups A and B post treatment revealed a significant decrease in Modified Arabic DASH-9 score of the group A compared with that of group $B(P=0.02)$ and a significant increase in hand grip strength of the group A compared with that of group B ( $\mathrm{P}=0.01)$ (Table 2).

Table 1. Basic characteristics of participants.

\begin{tabular}{|c|c|c|c|c|}
\hline \multicolumn{2}{|l|}{ Items } & $\begin{array}{c}\text { Group A } \\
\text { Mean } \pm \text { SD }\end{array}$ & $\begin{array}{c}\text { Group B } \\
\text { Mean } \pm \text { SD }\end{array}$ & P-value \\
\hline Age (years) & & $55.4 \pm 4.91$ & $55 \pm 5.61$ & 0.83 \\
\hline \multirow{2}{*}{$\operatorname{Sex}(n, \%)$} & Females & $1(7 \%)$ & $2(13 \%)$ & \multirow[b]{2}{*}{1} \\
\hline & Males & $14(93 \%)$ & $13(87 \%)$ & \\
\hline
\end{tabular}

SD, standard deviation; $\mathrm{p}$-value= level of significance

Table 2. Mean modified Arabic DASH score and hand grip strength pre and post treatment of the groups A \& B.

\begin{tabular}{|c|c|c|c|c|c|}
\hline Items & $\begin{array}{c}\text { Group A } \\
\text { Mean } \pm \text { SD }\end{array}$ & $\begin{array}{c}\text { Group B } \\
\text { Mean } \pm \text { SD }\end{array}$ & MD & T-value & P-value \\
\hline \multicolumn{6}{|c|}{ Modified Arabic DASH-9 score } \\
\hline Pre treatment & $26.73 \pm 5.2$ & $25.06 \pm 5.1$ & 1.67 & 0.88 & 0.38 \\
\hline Post treatment & $17.46 \pm 5.04$ & $22.26 \pm 5.67$ & -4.8 & -2.44 & 0.02 \\
\hline $\mathrm{MD}$ & 9.27 & 2.8 & & & \\
\hline$\%$ of change & 34.68 & 11.17 & & & \\
\hline T-value & 10.45 & 4.58 & & & \\
\hline P-value & $p=0.001$ & $p=0.001$ & & & \\
\hline \multicolumn{6}{|c|}{ Hand grip strength (lb) } \\
\hline Pre treatment & $15.73 \pm 2.43$ & $16.13 \pm 2.74$ & -0.4 & -0.42 & 0.67 \\
\hline Post treatment & $20.46 \pm 2.5$ & $17.86 \pm 3.13$ & 2.6 & 2.5 & 0.01 \\
\hline $\mathrm{MD}$ & -4.73 & -1.73 & & & \\
\hline$\%$ of change & 30.07 & 10.73 & & & \\
\hline T-value & -12.75 & -8.4 & & & \\
\hline P-value & $p=0.001$ & $p=0.001$ & & & \\
\hline
\end{tabular}

Group A= study group (the extracorporeal shockwave therapy); Group B= traditional physical therapy protocol (only received two sessions per week for 6 weeks involved ultrasound, massage splinting and stretching techniques); $\mathrm{SD}=$ standard deviation; $\mathrm{MD}=$ mean difference; $\mathrm{p}$ value $=$ probability value. 
Palmar fibromatosis is pathological condition which affects the palmar facia of the hand ensuing flexion disfigurements of the affected fingers. The earliest posted details and description of the problem was reported in 1614 by Felix Platter, yet it was Guillian Dupuytren who introduced a careful anatomic and obsessive investigation in 1831. What was extraordinary about palmar fibromatosis was that he accentuated the clinical course of the illness, the palmar fibromatosis influencing the hands frequently brings about constraints in aptitude and can fundamentally affect a person's capacity to participate in professional and a vocational exercises [15].

The condition is a significant reason for morbidity which diminishes their personal satisfaction, patients report feeling danger concerns, communicating dread that the fixed flexion of the finger will make it more defenseless to wounds. At the end, patients living with palmar fibromatosis additionally express worries about what their contracture restrict their social contact, mostly their capacity to give an appropriate handshake. Therefore, fixation of flex disfigurements is very important. Tragically, in the within side the lack a complete knowledge and comprehension of the molecular pathology, treatment is presently pointed toward disposing of the contracted pathologic disease tissue, without tending to the basic pathophysiology. As far as the best and the most widely recognized methodology is the surgical resection followed concentrated physical therapy program, in severe cases palmar fibromatosis may lead to amputation of the affected finger [7].

We found that one session of ESWT is an applicable and safe, and noninvasive can decrease palmar fibromatosis over a time of one month and half essentially. At this point, various gainful impacts of ESWT on different tissues have been accounted, for example, stem cell proliferation [16], growth factor stimulation [17] and anti-inflammatory actions via COX2-pathways [18]. Direct pain modulation via neuropeptides like calcitonin gene related peptide (CGRP) and substance P (SP) might explain part of the beneficial ESWT action in this trial [19]. Aykut et al. [8] revealed that ESWT in the early term can be chosen over the expensive infusion and surgical procedures as it expands the personal satisfaction and postpones the repeat of contractures. We accept a substantial treatment convention for ESWT must be set up with additional randomized controlled series with long term results and an absolute treatment for palmar fibromatosis still doesn't exist. Knobloch et al. [20] revealed that high energetic focused electromagnetic extracorporeal shockwave treatment (ESWT) is a successful and safe noninvasive treatment to decrease the pain in the early nodular phase of palmar fibromatosis. Three focused type of ESWT could show a considerable pain decrease and a supported impact more than year and a half of follow up in this randomized controlled trial. Abdulsalam et al. [6] reported that extracorporeal shock wave therapy effectively reduced pain and tenderness symptoms of patients with palmar fibromatosis, assesed the effectiveness of shock wave therapy on four patients by referring some of the items of the Michigan Hand Outcome Questionnaire. Brunelli et al. [7] announced that a low intensity ESWT (Li-ESWT) session might be done by physiotherapists in clinics with low cost in comparison with the surgical procedures, not harmful. It is by all accounts successful in the palmar fibromatosis treatment. Further investigations, including randomized controlled trials, are expected to assess the adequacy of outspread ESWT in palmar fibromatosis. Kumar et al. [21] reported that Shockwave treatment ends up being promising successful nonintrusive treatment in decrease of palmar fibromatosis.

\section{CONCLUSION}

It was concluded that extracorporeal shockwave therapy is a successful and effective treatment in reducing palmar fibromatosis.

\section{DECLARATIONS}

\section{Corresponding Author}

E-mail: mennasaad6917@gamil.com; ORCİD: 0000-0001-9045-7175

\section{Authors' contribution}

All authors contributed equally to this work.

\section{Competing interests}

The authors declare that they have no competing interests. 


\section{REFERENCES}

1. Henry M. Dupuytren's disease: current state of the art. Hand. 2014; 9(1):1-8. Article link I DOI: https://doi.org/10.1007\%2Fs11552-013-9563-0

2. RMafi, S Hindocha, and W Khan. Recent Surgical and Medical Advances in the Treatment of Dupuytren's Disease ASystematic Review of the Literature. The Open Orthopaedic Journal. 2012: 6(1):77-82 Article link | DOI: https://dx.doi.org/10.2174/1874325001206010077 I PMC3293169

3. Hurst LC, Badalamente MA. Nonoperative treatment of Dupuytren's disease. Pubmed Hand Clin. 1999 Feb; 15(1):97-107, vii. Hurst LC, Badalamente MA. Nonoperative treatment of Dupuytren's disease. Hand Clinics. 1999; 15(1):97-107. Article link I PMID: 10050246

4. Balaguer T, David S, Ihrai T, Cardot N, Daideri G, Lebreton E. Histological staging and Dupuytren's disease recurrence or extension after surgical treatment: a retrospective study of 124 patients. Journal of Hand Surgery (European Volume). 2009; 34(4):493-6. Article link I DOI : https://doi.org/10.1177\%2F1753193409103729

5. Chaussy $\mathrm{CH}$, Brendel W, Schmiedt E. Extracorporeally induced destruction of kidney stones by shock waves. The Lancet. 1980; 316(8207):1265-8. Article link | DOI : https://doi.org/10.1016/Sol40-6736(80)92335-1

6. Abdulsalam AJ, Shehab D, Elhady AA, Abraham M. High-energy focused extracorporeal shockwave therapy relieved pain in Dupuytren's disease: a series of seven hands. European Journal of Physical and Rehabilitation Medicine. 2018; 55(6):862-4. Article link | DOI : https://doi.org/10.23736/s1973-9087.18.05498-9 I PMID: 30370754

7. Brunelli S, Bonanni C, Traballesi M, Foti C (2020) Radial ESWT: a novel approach for the treatment of Dupuytren's contractures: a case report. Medicine (Baltimore) 99(24):e20587. Article link | PMC7302658 | DOI : https://dx.doi.org/10.1097\%2FMD.0000000000020587

8. Aykut S, Aydın C, Öztürk K, Arslanoğlu F, Kılınç CY. Extracorporeal Shock Wave Therapy in Dupuytren's Disease. The Medical Bulletin of Sisli Etfal Hospital. 2018; 52(2):124. Article link I PMCID: PMC7315057 | DOI : https://dx.doi.org/10.14744\%2FSEMB.2017.58076

9. Alsayed DG. Validation of the Arabic version of modified QuickDASH-9 scale to measure the quality of recovery after upper limb burn injury. CU Theses. 2012. Article link I Google Scholar

10. Spark T, Godlwana L, Ntsiea MV. Range of movement, power and pinch grip strength post flexor tendon repair. SA Orthopaedic Journal. 2018; 17(1):47-54. Article link | DOI: https://dx.doi.org/10.17159/2309-8309/2018/v17n1a7

11. Alexander LD, Gilman DR, Brown DR, Brown JL, Houghton PE. Exposure to low amounts of ultrasound energy does not improve soft tissue shoulder pathology: a systematic review. Physical Therapy. 2010; 90(1):14-25. Article link | DOI: https://doi.org/10.2522/ptj.20080272

12. Coverdale JJ. An editorial note on nomenclature: Orthosis versus splint. Journal of Hand Therapy. 2012; 25(1):3-4. Article link | DOI: https://doi.org/10.1016/j.jht.2011.10.002

13. Christie WS, Puhl AA, Lucaciu OC. Cross-frictional therapy and stretching for the treatment of palmar adhesions due to Dupuytren's contracture: a prospective case study. Manual Therapy. 2012; 17(5):479-82. Article link | DOI: https://doi.org/10.1016/j.math.2011.11.001

14. Nakano J, Yamabayashi C, Scott A, Reid WD. The effect of heat applied with stretch to increase range of motion: a systematic review. Physical Therapy in Sport. 2012; 13(3):180-8. DOI: https://doi.org/10.1016/j.ptsp.2011.11.003 | Article $\underline{\text { link }}$

15. Samulènas G, Rimdeika R, Braziulis K, Fomkinas M, Paškevičius R. Dupuytren's contracture: incidence of injury-induced cases and specific clinical expression. Medicina. 2020; 56(7):323. DOI: https://doi.org/10.3390/medicina56070323 । Article link

16. Cheng JH, Wang CJ, Chou WY, Hsu SL, Chen JH, Hsu TC. Comparison efficacy of ESWT and Wharton's jelly mesenchymal stem cell in early osteoarthritis of rat knee. American Journal of Translational Research. 2019; $11(2): 586$. Article link | Journal Issue | PMID: 30899364 | PMCID: PMC6413288

17. Hashimoto S, Ichinose T, Ohsawa T, Koibuchi N, Chikuda H. Extracorporeal shockwave therapy accelerates the healing of a meniscal tear in the avascular region in a rat model. The American Journal of Sports Medicine. 2019; 47(12):2937-44. Article link | DOI: https://doi.org/10.1177\%2F0363546519871059

18. Jeon SH, Zhu GQ, Kwon EB, Lee KW, Cho HJ, Ha US, Hong SH, Lee JY, Bae WJ, Kim SW. Extracorporeal shock wave therapy decreases COX-2 by inhibiting TLR4-NFאB pathway in a prostatitis rat model. The Prostate. 2019; 79(13):1498504. Article link I DOI: https://doi.org/10.1002/pros.23880

19. Ochiai N, Ohtori S, Sasho T, Nakagawa K, Takahashi K, Takahashi N, Murata R, Moriya H, Wada Y, Saisu T. Extracorporeal shock wave therapy improves motor dysfunction and pain originating from knee osteoarthritis in rats. Osteoarthritis and Cartilage. 2007; 15(9):1093-6. Article link | DOI: https://doi.org/10.1016/j.joca.2007.03.011

20. Knobloch $\mathrm{K}$, Hellweg M, Sorg H, Nedelka T. Focused electromagnetic high-energetic extracorporeal shockwave (ESWT) reduces pain levels in the nodular state of Dupuytren's disease-a randomized controlled trial (DupuyShock). Lasers in Medical Science. 2021 Jan 23: 1-1. Article link I DOI: https://doi.org/10.1007/s10103-021-03254-9

21. Kumar SS, Gangopadhyay S. Effect of Shockwave Therapy on Reduction of Dupuytren's Disease: A Case Report. Article link | Google scholar 